\title{
Efecto de selladores endodónticos sobre el pH del medio al cual son inmersos
}

\section{Effect of endodontic sealers on $\mathrm{pH}$ of the medium in which they are immersed}

\author{
Cecilia Inés Rourera ${ }^{1}$ (D) , Claudia Sotomayor ${ }^{2}$, Carolina Andrada Castillo ${ }^{1}$, Andrea Kaplan ${ }^{3}$, Gabriela \\ Martin $^{1,4}$. \\ 1 Universidad Católica de Córdoba. Facultad de Ciencias de la Salud. Carrera de Especialización de Endodoncia y odontología. \\ 2 Universidad Nacional de Córdoba. Facultad de Ciencias Químicas. Departamento de Bioquímica Clínica. Centro de Investigaciones en Bioquímica e \\ Inmunología (CIBICI) CONICET. \\ 3 Universidad de Buenos Aires. Facultad de Odontología. Cátedra de Materiales Dentales. \\ 4 Universidad Nacional de Córdoba y del Nordeste. Facultad de Odontología. Cátedra de Endodoncia. \\ Correspondencia: Gabriela Martín. Universidad Católica de Córdoba. Carrera de Endodoncia, Córdoba, Argentina. Email: ggmartin@hotmail.com
}

\section{Resumen}

INTRODUCCIÓN: El uso de selladores en la obturación endodóntica es fundamental para lograr un sellado tridimensional del conducto radicular. Según su composición, se presentan selladores a base de resina epóxica, como el AH Plus y de biocerámico, como Bio-C Sealer y BioRoot RCS, siendo considerados los últimos como materialesbioactivos, por su capacidad de promover una reacción biológica específica sobre el tejido receptor, por la liberación de calcio y $\mathrm{pH}$ alcalino

OBJETIVO: Evaluar, in vitro la capacidad de diferentes selladores endodónticos para modificar el pH de la solución en la cual son inmersos, en distintos períodos de tiempo.

MATERIALES Y METODOS: Se prepararon 15 probetas de sellador en un molde de plástico. Se dejaron fraguar 24 hs en estufa a $37^{\circ} \mathrm{C}$ y posteriormente, cada una fue inmersa en un frasco consolución fisiológica. La muestra se dividió en tres grupos ( $n=5$ ) según el sellador: 1) BioRoot RCS, 2) Bio-C Sealer y 3) AHPlus. Se determinó el $\mathrm{pH}$ de la solución de cada grupo en diferentes períodos de tiempo: inmediato, 40 min, 24 y 48 hs, 7, 14, 21 y 30 días. Los resultados fueron analizados estadísticamente mediante Análisis de Varianza con Medidas Repetidas (ANOVA).

RESULTADOS: El sellador AH Plus mantuvo constante el pH del medio durante toda la experiencia. Ambos selladores biocerámicos elevaron el pH del medio hasta llegar a ser alcalino $(11,2$ y 11,5) a las 24 hs, el cual se mantuvo para el grupo BioRoot RCS hasta los 30 días, con un pH promedio de 11.5. Mientras que, a partir de los 7 días, en el grupoBio-C Sealer comenzó a descender el pH del medio. La evolución del $\mathrm{pH}$ a lo largo del tiempo arrojó diferencias significativas entre los distintos selladores evaluados $(\mathrm{p}<0,001)$. CONCLUSIONES: Bajo las condiciones del presente estudio, AH Plus no modificó el pH del medio y ambos selladores biocerámicos elevaron el $\mathrm{pH}$, alcanzado su máximo valor de alcalinidad a los 7 días; a partir de los cuales, BioRoot RCS mantuvo el pH elevado del medio y con Bio-C Sealer se observó un ligero descenso del $\mathrm{pH}$ hasta los 30 días.

Palabras claves: pH, material biocerámico, sellador resinoso. 


\begin{abstract}
INTRODUCTION: The endodontic sealers are essential for three-dimensional root canal obturation. The sealers are based on epoxy resin, AH Plus and bioceramic sealers, Bio-C Sealer and BioRoot RCS, which are bioactive materials due to the ability to cause a specific biologic reaction in integration with the receptor tissue, with a pH of 12,5 .

OBJECTIVE: To evaluate in vitro, the capacity of different endodontic sealers to modify the $\mathrm{pH}$ of the solution in which they are immersed, at different time.

MATERIALS AND METHODS: Fifteen samples of sealers were prepared and placed into holes of blister packs, which set for 24 hours in store at 37 degrees Celsius. Then, each sample was immersed in sterile plastic bottles with 30 millimeters of physiological solution. Samples were divided into three groups $(\mathrm{n}=5)$ according to the sealer: 1) BioRoot RCS, 2) Bio-C Sealer and 3) AHPlus. The $\mathrm{pH}$ measurements were performed in different periods of time with a digital $\mathrm{pH}$ meter: immediate, 40 min, 24 and $48 \mathrm{hs}, 7,14,21$ and 30 days. Data were made by means of the Variance Analysis Test with repeated measurements.

RESULTS: The $\mathrm{pH}$ of AH Plus did not change during the experience. At $24 \mathrm{hs}$, both bioceramic sealers increased medium $\mathrm{pH}$ up to alkaline value, which was maintained with BioRoot sealer (mean 11,54) until 30 days. At 7 days, the $\mathrm{pH}$ of the medium with Bio-C Sealer started decreasing. Evolution of sealers $\mathrm{pH}$ along the time revealed statistical significant difference $(p<0,001)$.

CONCLUSION: AH Plus sealer did not modifie the $\mathrm{pH}$ of the medium Both bioceramic sealers increased the $\mathrm{pH}$, reaching to the maximum alkaline value at 7 days, which was maintained with BioRoot RCS and lightly decreased with Bio-C Sealer at 30 days.
\end{abstract}

KeyWords: Hydrogen $\mathrm{pH}$, bioceramic material, resin based sealer.

\title{
Introducción
}

La obturación del conducto radicular tiene como objetivo rellenar tridimensionalmente el conducto, sin espacios vacíos, para prevenir la microfiltración y reinfección, evitando el pasaje de microrganismos y fluidos desde la porción coronaria y apical hacia el conducto radicular y viceversa ${ }^{1}$.

Los materiales normalmente usados para la obturación endodóntica, pueden dividirse en una fase sólida y un medio cementante o sellador. Como núcleo sólido, la gutapercha es el material mundialmente aceptado, por ser biocompatible y estable en el tiempo; sin embargo, carece de propiedades adherentes a la pared dentinaria, por lo que es necesario el uso de sellador. Según Grossman, un sellador ideal debe ser radiopaco, bacteriostático, insoluble frente a los fluidos tisulares, presentar adherencia a la pared dentinaria y conos de gutapercha, y ocupar el espacio a donde no llega el material de núcleo sólido²

Los selladores deben cumplir con requerimientos físicos, químicos y biológicos, dentro de los cuales son importantes sus propiedades antimicrobianas y biocompatibilidad con los tejidos perirradiculares. Por sus propiedades físicas de sellado, impiden el desarrollo de los microrganismos en los túbulos dentinarios y conducto radicular; pero, además, por su composición química y liberación de componentes, pueden modificar el $\mathrm{pH}$ del medio, impidiendo el crecimiento microbiano ${ }^{3}$.

Los selladores a base de óxido de zinc y eugenol han sido los más utilizados a nivel mundial por su adecuada plasticidad, consistencia, eficacia en el sellado y mínimas alteraciones volumétricas que presentan posterior al fraguado ${ }^{4}$. Sin embargo, se ha demostrado la toxicidad de estos selladores en contacto con tejidos vivos, generando una respuesta inflamatoria de leve a severa en los tejidos periapicales ${ }^{5}$. En otro aspecto, el eugenol interfiere en la polimerización de los materiales resinosos de restauración coronaria, que se utilizan posterior al tratamiento endodóntico ${ }^{6}$.

Los selladores a base de resina epóxica, que no contienen eugenol, han sido introducidos en la práctica endodóntica por sus ventajas de adhesión a la estructura dentaria, adecuado tiempo de trabajo, fácil manipulación y buen sellado ${ }^{7}$.AHPlus® (Dentsply Mailleffer, Suiza) es un sellador compuesto por resina epóxica y aminas, introducido en el mercado en 1997. Se ha demostrado que AH Plus ${ }^{\circledR}$ presenta adecuado sellado, estabilidad dimensional, alta radiopacidad y polimerización sin formación de formaldehído y propiedades autoadhesivas ${ }^{8}$.

En 1993 se desarrolló el primer cemento biocerámico a base de trióxido mineral agregado MTA (ProRoot Dentsply, Suiza) para el sellado de perforaciones accidentales del conducto radicular ${ }^{9}$. MTA es un cemento bioactivo con capacidad para inducir la formación de nuevo tejido mineralizado, lo que hace que este material sea biológicamente aceptable para retrobturaciones en cirugías apicales, y para el tratamiento de apexificación y endodoncia regenerativa en dientes con ápices 
inmaduros ${ }^{10}$. Su mecanismo de acción se basa en la liberación de iones calcio que activan la adhesión, proliferación celular, y por su $\mathrm{pH}$ elevado se crea un medio antibacteriano ${ }^{11}$. Posteriormente, aparecieron en el mercado otras formas comerciales, como MTA Angelus $®$, con similares propiedades químicas al MTA conteniendo bismuto para aumentar su radiopacidad $^{12}$.En el año 2009 se presentó Biodentine ${ }^{\circledR}$ (Septodont, SaintMaur-des-Fosses, Francia) un nuevo cemento bioactivo, sustituto de la dentina, compuesto principalmente por silicato tricálcico ${ }^{13}$. Por sus propiedades biológicas y físicas mejoradas, Biodentine presenta una resistencia a la compresión significativamente mayor que MTA White ${ }^{14}$. Además, su microdureza, resistencia a la flexión, capacidad de sellado, fuerza de adhesión y liberación de iones de calcio son notablemente superiores en comparación con otros cementos a base de silicato tricálcico ${ }^{15}$. Una de las ventajas de Biodentine es que el tiempo de fraguado es de 15 minutos, resultando más corto respecto al del MTA que fragua en 4 horas (Darvell y Wu 2011) y su estabilidad del color en el tiempo ${ }^{16}$.

Posteriormente, se desarrollaron los selladores a base de biocerámicos, indicados para la obturación permanente del conducto radicular en combinación con conos de gutapercha, mediante la técnica de cono único o compactación lateral en frío13. BioRoot RCS (Septodont, SaintMaur-desFosses, Francia) se presenta en forma de polvo y líquido. El polvo contiene óxido de circonio como radiopacificador y un polímero biocompatible hidrofílico para mejorar la adherencia. El líquido contiene principalmente agua y cloruro de calcio lo cual acelera el fraguado. BioRoot RCS mostró un sellado hermético con la dentina y la gutapercha, y una radiopacidad adecuada ${ }^{17}$.Se ha demostrado que los selladores biocerámicos son bioactivos porque estimulan el proceso fisiológico del hueso y la mineralización de la estructura dentinaria ${ }^{18}$. Por sus propiedades bioactivas tales como la biocompatibilidad, la formación de hidroxiapatita, mineralización de la estructura dentinaria, $\mathrm{pH}$ alcalino y propiedades de sellado; se crea un entorno favorable para la cicatrización periapical $^{19}$.

Recientemente, apareció en el mercado Bio-C Sealer (Angelus, Brasil), un sellador biocerámico que contiene silicato de calcio, aluminato de calcio y óxido de calcio, lo cual hace que este material sea biocompatible y bioactivo, por la liberación de iones calcio. Además, contiene óxido de zirconio, óxido de hierro, dióxido de silicio y propilenglicol como agente de dispersión, sin sufrir contracción posterior al fraguado Las propiedades mecánicas y físicas confieren facilidad en el manejo y sellado hermético de los conductos, además de sus propiedades biológicas y $\mathrm{pH}$ elevado 12,5$)^{20}$.

\section{Objetivo}

El objetivo del presente estudio fue evaluar in vitro la capacidad de diferentes selladores endodónticos para modificar el $\mathrm{pH}$ de la solución en la cual fueron inmersos, en distintos períodos de tiempo.

\section{Material y métodos}

Tipo de Investigación: Experimental

Características generales: La presente investigación se desarrolló en la Carrera de Endodoncia y en el Laboratorio de Química de la Facultad de Ciencias de la Salud de la Universidad Católica de Córdoba.

\section{Método y diseño}

Se seleccionaron tres selladores endodónticos: uno a base de resina epóxica AH Plus (Dentsply) y dos a base de biocerámicos, BioRoot RCS (Septodont) y Bio-C-Sealer (Angelus). Se prepararon 15 muestras de sellador, siguiendo las indicaciones del fabricante, y se colocaron en un molde de plástico, obteniendo muestras de $8 \mathrm{~mm}$ de diámetro por $3 \mathrm{~mm}$ de profundidad. Para su fraguado, se conservaron a $37^{\circ} \mathrm{C}$ durante $24 \mathrm{hs} \mathrm{y}$ posteriormente, cada muestra fue inmersa en un frasco estéril de plástico con $30 \mathrm{ml}$ de solución fisiológica estéril. Las muestras se dividieron en 3 grupos $(n=5)$, según el sellador utilizado, quedando conformados de la siguiente manera:

Grupo 1: BioRoot RCS (Septodont)

Grupo 2: Bio-C-Sealer (Angelus)

Grupo 3: AHPlus (Dentsply)

Se realizaron las mediciones de $\mathrm{pH}$ de la solución de cada frasco, por duplicado, con un peachímetro (LT Lutron de HANNA), en los diferentes períodos de tiempo: inicial (inmediatamente después de la inmersión en la solución fisiológica), a los 40 minutos, a las 24 hs, 72hs, 7, 14, 21 y 30 días. Al momento de estandarización de la técnica, se evaluó el pH del medio en ausencia de sellador y no se observaron cambios en la cinética de tiempos evaluados. Durante los 30 días, las muestras fueron conservadas a temperatura ambiente, en el Laboratorio de Química de la Facultad de Ciencias de la Salud de la Universidad Católica de Córdoba, a cargo del Dr. Agustín Joison. 


\section{Análisis estadístico}

Los datos fueron evaluados mediante análisis de varianza con medidas repetidas, utilizando como factor el sellador, el efecto tiempo y la interacción entre el tiempo y sellador. Para tales análisis, se utilizó el software SPSS y un nivel de significación de $(\mathrm{P}<0,05)$.

\section{Consideraciones éticas}

El estudio se realizó cumpliendo con la normativa de la Declaración de Helsinki, Buenas Prácticas Clínicas de ANMAT y Ley Provincial de investigación en salud $\mathrm{N}^{\circ}$ 9694. Se asegura protección de datos personales de los pacientes según la Ley 25.326. Los autores declaran no tener conflictos de intereses.

\section{Resultados}

En la (Tabla 1 ) se muestran los valores promedio de las mediciones de $\mathrm{pH}$ de la solución fisiológica donde estaban inmersos los selladores, a los diferentes períodos de tiempo en lo que fue evaluado. La evolución del $\mathrm{pH}$ a lo largo del estudio cinético reveló diferencias significativas entre los selladores $(p<0,001)$, siendo BioRoot RCS el sellador que provocó un mayor incremento del $\mathrm{pH}$, con un promedio de 11.5 (Tabla 2). Las variaciones del $\mathrm{pH}$ del medio en función de la cinetica del tiempo fue estadísticamente significativa $(\mathrm{p}<0,001)$.

Tabla 1. Valores promedio de $\mathrm{pH}$ para cada sellador en los diferentes tiempos.

Letras diferentes implican diferencias significativas.

\begin{tabular}{|l|c|c|c|}
\hline $\begin{array}{l}\text { Períodos de } \\
\text { tiempos }\end{array}$ & AH Plus & $\begin{array}{c}\text { Bio-C } \\
\text { Sealer }\end{array}$ & $\begin{array}{c}\text { BioRoot } \\
\text { RCS }\end{array}$ \\
\hline Inicial & 5,7 & 5,9 & 5,5 \\
& $\mathrm{a}$ & $\mathrm{a}$ & $\mathrm{a}$ \\
\hline $\mathbf{4 0}$ minutos & 6,7 & 10,7 & 10 \\
& $\mathrm{a}$ & $\mathrm{c}$ & $\mathrm{b}$ \\
\hline $\mathbf{2 4}$ hs & 5,8 & 11,2 & 11,5 \\
& $\mathrm{a}$ & $\mathrm{b}$ & $\mathrm{c}$ \\
\hline $\mathbf{4 8}$ hs & 6,6 & 11,1 & 11,8 \\
& $\mathrm{~s}$ & $\mathrm{~b}$ & $\mathrm{c}$ \\
\hline 7 días & 6,3 & 11,5 & 12,7 \\
& $\mathrm{a}$ & $\mathrm{b}$ & $\mathrm{c}$ \\
\hline 14 días & 6,2 & 9,7 & 11,8 \\
& $\mathrm{a}$ & $\mathrm{b}$ & $\mathrm{c}$ \\
\hline 21 días & 6,3 & 8,9 & 11,5 \\
& $\mathrm{a}$ & $\mathrm{b}$ & $\mathrm{c}$ \\
\hline 30 días & 6,4 & 8,2 & 11,2 \\
& $\mathrm{a}$ & $\mathrm{b}$ & $\mathrm{c}$ \\
\hline
\end{tabular}

Tabla 2. Valores de $\mathrm{pH}$ promedio del medio con los diferentes selladores.

DS: desviación estándar. Diferencias: letras diferentes implican diferencias significativas

\begin{tabular}{|l|l|l|l|}
\hline Sellador & Media general & DS & Diferencias \\
\hline $\begin{array}{l}\text { BioRoot } \\
\text { RCS }\end{array}$ & 11.54 & $+/-0.51$ & $\mathrm{a}$ \\
\hline Bio-C-Sealer & 10.22 & $+/-0.40$ & $\mathrm{~b}$ \\
\hline AH Plus & 6.39 & $+/-0.15$ & $\mathrm{c}$ \\
\hline
\end{tabular}

El pH de AH Plus se mantuvo constante durante la experiencia y tanto Bio-C Sealer como BioRoot RCS incrementaron el $\mathrm{pH}$ a partir de los $40 \mathrm{~min}$. Cabe destacar, que a los $40 \mathrm{~min}$. Bio-C Sealer elevó significativamente el $\mathrm{pH}$ del medio, pero a las $24 \mathrm{hs}$ y hasta el final de la experiencia los valores observados fueron significativamente más bajos que los observados para BioRoot RCS. A su vez, el máximo valor de $\mathrm{pH}$ en los selladores biocerámicos se observó a los 7 días y luego fue parcialmente declinando, aunque más velozmente en las muestras que recibieron tratamiento con Bio-C Sealer que en las que fueron tratadas con BioRoot RCS. A partir de los 7 días se observó mayor variabilidad en la respuesta (Figura 1)

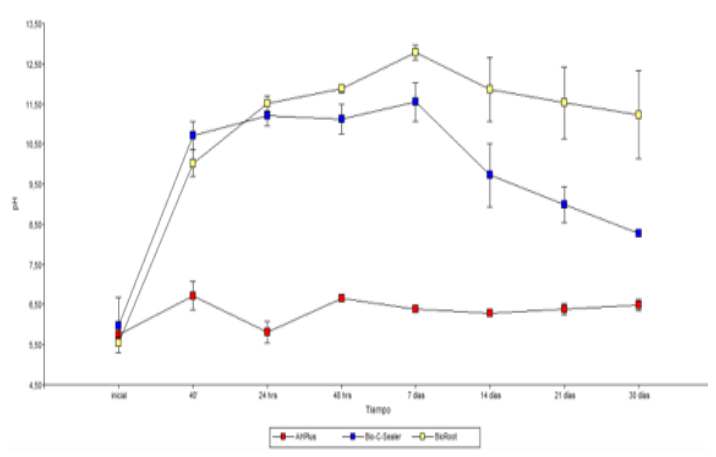

Figura 1: Mediciones de $\mathrm{pH}$ del medio con los selladores AH Plus, Bio-C Sealer y BioRoot RCS en el tiempo.

\section{Discusión}

El uso de selladores en la obturación endodóntica es fundamental para lograr un sellado tridimensional del conducto radicular ${ }^{2}$. Según su composición, se presentan selladores a base de resina epóxica, como el AH Plus y de biocerámico, como Bio-C Sealer y BioRoot RCS, siendo considerados los últimos como materiales bioactivos, por su capacidad de promover una reacción biológica específica sobre el tejido receptor, por la liberación de calcio y $\mathrm{pH}$ alcalino $^{18,20}$. En un estudio realizado por Siboni $\mathrm{F}$ $\mathrm{y} \mathrm{col}^{21}$ confirmaron que BioRoot RCS libera iones 
$\mathrm{Ca}++$, une iones biológicamente activos y activa la nucleación de precursores de hidroxiapatita en la superficie del material. Por otro lado, AH Plus no mostró la capacidad de liberar iones, entonces la formación de depósitos de fosfatos de calcio estuvo relacionado a la presencia de grupos funcionales en el material que absorben los iones involucrados en la nucleación de hidroxiapatita. De este modo, quedó demostrado que BioRoot RCS es un material bioactivo capaz de nuclear los depósitos de apatita carbonatada en relación a su capacidad prolongada de liberar iones calcio y de alcalinizar el medio ${ }^{22}$. Estos datos apoyarían el potencial bioactivo de los selladores de silicato tricálcico como BioRoot RCS de promover la salud periapical, la regeneración ósea y sellar por mineralización y depósitos de hidroxiapatita en la interfaz de la pared del conducto radicular. Estos hallazgos concuerdan con nuestro estudio donde se observó que los selladores biocerámicos BioRoot RCS y Bio-C-Sealer alcalinizaron el medio.

Por su parte, Molgatini y col ${ }^{23}$. demostraron que el sellador AH Plus no posee la capacidad de liberar sales ni óxidos como en otros selladores analizados en su experiencia; ni iones que alcalinicen el medio. Del mismo modo, en nuestro estudio el sellador a base de resina epoxi AHPlus no modificó el $\mathrm{pH}$ del medio y se mantuvo un $\mathrm{pH}$ neutro en todos los períodos de tiempo.

En un estudio realizado por Zordan-Bronzel y col ${ }^{20}$ concluyeron que Bio-C-Sealer presenta menor tiempo de fraguado que BioRoot RCS (60 a 120 minutos para Bio-C Sealer versus 500 minutos para BioRoot RCS) y tiene la capacidad de alcalinizar el medio. En este estudio, las muestras fueron sumergidas en agua desionizada y se realizaron mediciones con un peachímetro digital a los 7, 14 y 21 días; comparando Bio-C Sealer con el sellador biocerámico Total Fill BC Sealer (FKG Dentaire SA, La Chaux-de-Fonds, Switzerland) y con sellador AH Plus (Dentsply). El pH fue más alto para el TotalFill el primer día, pero después no hubo diferencia significativa a los 7,14 y 21 días. Los mismos autores, en un estudio previo ${ }^{24}$ demostraron que el $\mathrm{pH}$ elevado (12) confiere a los cementos y selladores biocerámicos acción antibacteriana; y que la reacción de fraguado en los materiales biocerámicos ocurre en presencia de humedad, ya que son materiales con características hidrofílicas. Por lo anteriormente expuesto, estos materiales deberían ser usados en la práctica clínica, en conductos ligeramente húmedos para absorberla humedad de los fluídos presentes en los túbulos dentinarios y en los tejidos periapicales. La solubilidad de un sellador indica la pérdida de masa de material cuando es inmerso en agua ${ }^{4}$. El $\mathrm{pH}$ elevado de los biocerámicos y su poder alcalinizante estaría dado por la liberación de iones $\mathrm{Ca}++$ y $\mathrm{OH}$ - gracias a la solubilidad de los mismos en el medio líquido al cual son inmersos ${ }^{25}$. $\mathrm{AH}$ Plus mantiene estable y modifica escasamente el $\mathrm{pH}$ debido a fuertes enlaces cruzados en materiales a base de resina epoxi que los hace de baja solubilidad $^{26}$.

En un estudio realizado por Urban y col. ${ }^{27}$ demostraron que los valores de $\mathrm{pH}$ fueron superiores cuando fueron probados en agua destilada que en PBS. Bajo un punto de vista clínico, debe especularse que los valores de $\mathrm{pH}$ medidos en PBS similar al fluido corporal son más realistas que aquellos obtenidos en agua destilada. Este estudio que duró 6 meses, reveló que en agua destilada el sellador BioRoot RCS mantuvo un $\mathrm{pH}$ alcalino durante todo el período del experimento, mientras que $\mathrm{AH}$ Plus tuvo un $\mathrm{pH}$ ligeramente ácido y posterior a los 4 meses se mantuvo neutro hasta el final del estudio. En PBS, BioRoot RCS reveló un $\mathrm{pH}$ alcalino por 4 meses y $\mathrm{AH}$ Plus neutro. Por otro lado, también encontraron a los 14 días, sobre la superficie de todos los especímenes de BioRoot inmersos en PBS, un precipitado blanco cuyo principal componente químico fue hidroxiapatita. Presumiblemente, estos autores sostienen que por esta precipitación la solubilidad del BioRoot RCS decrece. Esta solubilidad fue significativamente más alta que en el sellador convencional de resina AH Plus, en el cual no pudo ser determinado ningún precipitado. Según estos autores, BioRoot RCS no incluye una fase de fosfato de Calcio, sin embargo, esta fase fue identificada cuando el material fue inmerso en solución fisiológica o en solución bufferizada salina de Hank o en PBS. Este depósito parece impedir un aumento adicional de la solubilidad. Por lo tanto, se puede esperar que si un silicato de calcio que contiene el sellador es inmerso en un líquido simulando fluido corporal, los iones de calcio combinados con fosfatos están promoviendo la formación de una capa superficial de hidroxiapatita, la cual es capaz de llenar espacios vacíos originados por la alta solubilidad $^{20}$.El calcio, el silicio y el carbonato difunden en los túbulos dentinarios, lo que resulta en la formación de una "zona de infiltración marginal", otorgando alta resistencia a las microfiltraciones y anclaje micromecánico mediante la precipitación en la entrada de los túbulos dentinarios. Al igual que otros cementos biocerámicos, no se contraen, sino que posee una pequeña expansión durante su fraguado ${ }^{26}$. Por lo tanto, si los vacíos se reducen con el tiempo, como fue demostrado in vitro, los selladores a base de silicato de calcio en combinación con gutapercha podrían mejorar la capacidad del sellado por el depósito de hidroxiapatita (fosfato de calcio) en la interfase sellador pared de dentina ${ }^{25}$. Sin embargo, si bien la solubilidad pareciera ser responsable de la liberación de iones alcalinizantes con la

Revista Methodo: Investigación Aplicada a las Ciencias Biológicas. Universidad Católica de Córdoba. Jacinto

Ríos 571 Bo Gral. Paz. X5004FXS. Córdoba. Argentina. Tel.: (54) 3514517299 / Correo: methodo@ucc.edu.ar

/ Web: methodo.ucc.edu.ar | ARTICULO ORIGINAL Rev. Methodo 2021;6(1):13-19. 
consecuente elevación de $\mathrm{pH}$, como fue demostrado por Urban y $\operatorname{col}^{27}$ y en el presente estudio, sigue siendo un importante desafío que requiere futuras investigaciones.

\section{Conclusión}

Bajo las condiciones del presente estudio, el sellador AH Plus no modificó el pH del medio y se mantuvo estable. Ambos selladores biocerámicos elevaron el $\mathrm{pH}$, alcanzando su máximo valor de alcalinidad a los 7 días; a partir de los cuales, BioRoot RCS mantuvo el pH elevado del medio y con Bio-C Sealer se observó un ligero descenso del pH hasta los 30 días.

\section{Bibliografía}

1. Johnson W, Kulildjc C, Tayf K. Obturation of the cleaned and shaped root canal system. In Hargreaves KH, Berman LH, eds. Cohen Pathway of the Pulp, 11th ed. St Louis: Elsevier 2016; 280-323.

2. Suresh G, Gopikrishna V. Obturation of the radicular space. In: Suresh Chandra B, Gopikrishna V, EDS. Grossman's endodontic Practice, 13th Ed. New Delhi: Wolters Kluwer Health 2014; 343-73.

3. Hargreaves K, Cohen S. Instrumental and Device Canal Filling. 10th Ed.Elsevier 2011; 260-264.

4. Versiani MA, Abi Rached-Junior FJ. Zinc oxide nanoparticles enhance physicochemical characteristics of Grossman sealers. J Endod 2016; 42: 1804-10.

5. Araki K, Suda H, Spangberg L. Indirect longitudinal cytotoxicity of root canal sealers L929 cells and human periodontal ligament fibroblasts. J. Endod. 1998; 20 (1): 67-70.

6. Marín-Bauza GA, Silva-Sousa TC, Cunha SA et al. Physicochemical properties of endodontic sealers of different bases. J Appl Oral Sci 2012; 20(4):455-461.

7. ResendeLM, RachedFJ, Versiane MA et al.A comparative study of physicochemical properties of AH Plus, Epiphany, and Epiphany SE root canal sealers. IntEndod J 2009;42(9):785-793.

18. Camps J, Jeanneau C, Elayachi I, Laurent P, About I. Bioactivity of a calcium silicatebased endodontic cement (BioRoot RCS): interactions with human periodontal ligament cells in vitro. J Endod 2015; 41(9):1469-73
8. Hubbe L, Oliveira D, Vianna K, Coelho S. AH Plus extrusion into periapical tissue: literature 74 review of main related properties and report of clinical cases. RSBO RevistaSulBrasileira de Odontologia 2016;13(4): 280 288.

9. Lee S, Monsef M, Torabinejad M. Sealing ability of mineral trioxide aggregate for repair of lateral root perforations. J Endod1993; 19: 541-4

10. Bakland L, Andreasen J. Will mineral trioxide aggregate replace calcium hydroxide in treating pulpal and periodontal healing complications subsequent to dental trauma? A review. Dent Traumatol. 2012; 28: 25-32.

11. Porter M, Bertó A, Primus C, Watanabe I. Physical and chemical properties of newgeneration endodontic materials. J Endod2010; 36(3):524-8.

12. Oliveira M, Xavier C, Demarco F, Pinheiro A et al.Comparative chemical study of MTA and portland cements. Braz Dent J 2007; 18(1): 37

13. CamillieriJ, Sorrentino F, Damidot D.Investigation of the hydration and bioactivity of radiopacified tricalcium silicate cement, Biodentine and MTA Angelus. Dental Materials 2013, 29(5): 580-593.

14. ElnaghyAM. 2014. Influence of Acidic Environment on Properties of Biodentine and White Mineral Trioxide Aggregate: A Comparative Study. J Endod 2014; 40(7): 953-957.

15. Rajasekharan S, Martens L, Cauwels R, Anthonappa R. Biodentine material characteristics and clinical applications: a 3year literature review and update. Eur Arch Paediatr Dent 2018; 19(1):1-22.

16. Kohli MR, Yamaguchi M, SetzerFC, Karabucak B. Spectrophotometric analysis of coronal tooth discoloration induced by various bioceramic cements and other endodontic materials. J Endod 2015; 41(11): 1862-1866.

17. Xuereb M, Vella P, Damidot D, Sammut CV, Camilleri J. In Situ assessment of the setting of tricalcium silicate-based sealers using a dentin pressure model. J Endod 2015; 41(1):111-124.

19. Dimitrova-NakovS, Uzunoglu E, ArdilaOsorio H, Baudry A et al.In vitro bioactivity of Bioroot ${ }^{\mathrm{TM}} \mathrm{RCS}$, via A4 mouse pulpal stem cells. Dental Materials 2015, 31(11): 1290-7 
20. Zordan-Bronzel C, Estevez Torres F, Tonomaru-Filho M, Chavez-Andrade $\mathrm{G}$ et al. Evaluation of physicochemical properties of a new calcium cilicate-based sealer, Bio-CSealer. J Endod 2019; 45:1248-1252.

21. Siboni F, Tadedei P. Properties of BioRoot RCS, a tricalcium silicate endodontic sealer modified with povidone and policarboxylate. IntEndod J 2017; 50: 20-36.

22. Prullage RK, Urban K. Material properties of a tricalcium silicate containing, and epoxy resin-based root canal sealers. J Endod 2016; 42: $1784-8$.

23. Molgatini S, Perez S.Tejerina D, La Rosa A, Kaplan A. Changes of $\mathrm{pH}$ produced by inmersion of endodontic sealers. ActaOdontolLatinoam 2002; 15: 15-19.

24. Zordan-Bronzel CL, Tanomaru-Filho M, Rodriguez EM, Chaves Andrade G et al. Cytocompatibility, bioactive potential and antimicrobial activity of an experimental calcium silicate-based endodontic sealer. IntEndod J. 2019; 52: 979-986.

25. Borges RP, Sousa-Neto. Changes in the surface of four calcium silicate-containig endodontic materials and epoxy resin-based sealers after a solubility test. IntEndodo $\mathbf{J}$ 2012; 45:419-28.

26. Viapiana R, Flumignan DL. Physicochemical and mechanical properties of zirconium oxide and niobium oxide modified Portland cementbased experimental endodontic sealers. IntEndod J 2014; 47:437-48.

27. Urban K, Neuhaus J, Donnermeyer D. Solubility and $\mathrm{pH}$. Value of 3 different root canal sealer: along-term investigation. $\mathbf{J}$ Endod 2018; 44: 1736-1740.

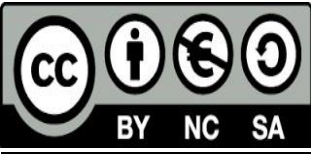

